FERMILAB-Conf-96/324

REVISION

\title{
Neutrino Radiation Hazards: A Paper Tiger
}

J. Donald Cossairt, Nancy L. Grossman and Elaine T. Marshall

Fermi National Accelerator Laboratory

P.O. Box 500, Batavia, Illinois 60510

September 1996

Submitted to Health Physics Society 1997 Midyear Topical Meeting,

San Jose, California, January 5-8, 1997 


\section{Disclaimer}

This report was prepared as an account of work sponsored by an agency of the United States Government. Neither the United States Government nor any agency thereof, nor any of their employees, makes any warranty, expressed or implied, or assumes any legal liability or responsibility for the accuracy, completeness, or usefulness of any information, apparatus, product, or process disclosed, or represents that its use would not infringe privately owned rights. Reference herein to any specific commercial product, process, or service by trade name, trademark, manufacturer, or otherwise, does not necessarily constitute or imply its endorsement, recommendation, or favoring by the United States Government or any agency thercof. The views and opinions of authors expressed herein do not necessarily state or reflect those of the United States Government or any agency thereof.

\section{Distribution}

Approved for public release; further dissemination unlimited. 


\author{
Neutrino Radiation Hazards: A Paper Tiger \\ J. Donald Cossairt, Nancy L. Grossman, and Elaine T. Marshall \\ Fermi National Accelerator Laboratory \\ P. O. Box 500 \\ Batavia, IL $60510^{*}$
}

\begin{abstract}
Neutrinos are present in the natural environment and are also produced by particle accelerators. A recent hypothesis has also been proposed that asserts that ionizing radiation due to neutrinos from certain astronomical events may have led to the extinction of some biological species. Thus, it is of interest to be able to estimate the dose equivalent due to these weakly interacting particles. Presented here are methods for estimating the dose equivalent due to neutrinos over a broad domain of energy, examples of such calculations, and an assessment of the postulated role of neutrinos in biological extinctions. It is concluded that the dose equivalent due to neutrinos from natural sources and from present-day accelerators is inconsequential and the postulated role of neutrinos in biological extinctions is highly improbable.
\end{abstract}




\section{INTRODUCTION}

Neutrinos are present in the natural environment due to terrestrial, solar, and cosmic sources and are also produced at accelerators both incidentally and intentionally as part of physics research programs. Progress in fundamental physics research has led to the creation of beams of neutrinos of ever-increasing intensity and/or energy. The large size and cost associated with these beams attracts, and indeed requires, public interest, support, and some understanding of the "exotic" particles produced, including the neutrinos. Furthermore, the very word neutrino ("little neutral one", as coined by Enrico Fermi) can possibly lead to public concern due to confusion with "neutron", a word widely associated with radiological hazards. Adding to such possible concerns is an assertion, widely publicized, that neutrinos from astronomical events may have led to the extinction of some biological species. Presented here are methods for conservatively estimating the dose equivalent due to neutrinos as well as an assessment of the possible role of neutrinos in biological extinction processes.

\section{NEUTRINO INTERACTIONS AND FLUENCE-TO-DOSE EQUIVALENT CONVERSION FACTORS}

Neutrinos ( $\left.v^{\prime} s\right)$ are neutral leptons, conventionally believed to be massless, that interact with matter solely through the weak interaction with very small cross sections. The Standard Model of Electroweak Interactions of Weinberg, Salam, and Glashow (Langacker and Erler 1996), highly successful in explaining these interactions, underlies the work presented in this section. There are three known "flavors" of neutrinos and their corresponding antiparticles (antineutrinos, $\bar{v}^{\prime}$ s) so that there are six types of these particles altogether: electron neutrinos $\left(v_{\mathrm{e}}, \overline{v_{\mathrm{e}}}\right)$, muon neutrinos $\left(v_{\mu}, \overline{v_{\mu}}\right)$, and tau neutrinos $\left(v_{\tau}, \overline{v_{\tau}}\right)$.

In the present work, a tissue composition is assumed that includes all elements present in the whole body at the level exceeding one per cent by weight [hydrogen (10.1\%), carbon 
(23.2\%), nitrogen (2.6\%), oxygen (61.6\%), phosphorus (1.1\%), and calcium (1.4\%)] at a whole body density, $\rho$, of $1.07 \mathrm{~g} \mathrm{~cm}^{-3}$ (ICRP 1975). The average number densities of the atomic electrons, nucleons, and nuclei can be calculated, respectively; $\rho_{\text {electrons }}=3.54 \times 10^{23}$ $\mathrm{cm}^{-3}$, $\rho_{\text {nucleons }}=6.44 \times 10^{23} \mathrm{~cm}^{-3}$, and $\rho_{\text {nuclei }}=4.60 \times 10^{22} \mathrm{~cm}^{-3}$. The averages of atomic number, $\mathrm{Z}_{\mathrm{ave}}$, neutron number, $\mathrm{N}_{\mathrm{ave}}$, and atomic weight, $\mathrm{A}_{\mathrm{ave}}$, are $7.048,6.959$, and 14.007, respectively. The whole body is approximated by a tissue slab $30 \mathrm{~cm}$ thick.

\section{Process A: Neutrino scattering from atomic electrons}

Electrons recoiling from elastic scattering of neutrinos deposit energy in tissue. The cross section for this process, $\sigma_{v}$-electron, has been presented by Langacker and Erler (1996) and Bahcall (1989) as

$$
\sigma_{v \text {-electron }}=\mathrm{CE}_{v} \times 10^{-45}\left(\mathrm{~cm}^{2}\right),
$$

where $\mathrm{C}\left(v_{\mathrm{e}}\right)=9.2, \mathrm{C}\left(\overline{v_{\mathrm{e}}}\right)=3.9, \mathrm{C}\left(v_{\mu}\right)=1.6$, and $\mathrm{C}\left(\overline{v_{\mu}}\right)=1.3$ with $\mathrm{E} v$, the neutrino energy, in $\mathrm{MeV}$. The maximum recoil kinetic energy, $\mathrm{T}_{\max }\left(\mathrm{E}_{v}\right)$, that can be transferred by a massless particle of energy $E_{v}$ in an elastic collision with a particle of rest energy $m_{0} c^{2}$ is

$$
\mathrm{T}_{\max }=\frac{2 \mathrm{E}_{\mathrm{v}}^{2}}{\mathrm{~m}_{\mathrm{o}} \mathrm{c}^{2}+2 \mathrm{E}_{v}}
$$

A reasonable estimate of the average value of the recoil kinetic energy, $T_{a v e}$, is $0.67 \mathrm{~T}_{\max }$. Because of the large multiple scattering of the recoiling electrons, and the fact that their radiation quality factor is unity over a very large domain of energy, the fluence-to-dose equivalent conversion factor, $\mathrm{P}\left(\mathrm{E}_{\mathrm{v}}\right)$, for the neutrinos can be calculated as follows, with $\mathrm{T}_{\text {ave }}$ in $\mathrm{MeV}$ :

$$
\mathrm{P}\left(\mathrm{E}_{v}\right)=1.6 \times 10^{-4} \sigma_{v-\text { electron }} \mathrm{T}_{\text {ave }} \frac{\rho_{\text {electrons }}}{\rho}\left(\mu \mathrm{Sv} \mathrm{cm}^{2}\right) .
$$




\section{Process B: Neutrino scattering from nuclei}

In this process, described by Drukier and Stodolsky (1984), the neutrinos interact with nuclei coherently. The process is strictly effective for low energies where the de Broglie wavelength of the neutrinos is too long to resolve individual nucleons. At higher energies, scattering from nucleons (see Process C) gradually becomes more important. Here, the recoiling particles are low energy ions having large linear energy transfer (LET). Where strict coherence applies the cross section for this process is

$$
\sigma_{v-\text { nucleus }}=4.2 \times 10^{-45} \mathrm{~N}^{2} \mathrm{E}_{v}^{2}\left(\mathrm{~cm}^{2}\right)
$$

where $\mathrm{E}_{\mathrm{v}}$ is in $\mathrm{MeV}$ and $\mathrm{N}$ is the neutron number of the recoiling nucleus. For $\mathrm{E}_{\mathrm{V}}>20 \mathrm{MeV}$,

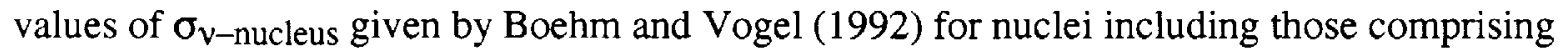
tissue were used. This process is considered to be independent of flavor, but the cross sections for $\bar{v}^{\prime}$ 's are approximately $1 / 2$ as large. $P\left(E_{v}\right)$, using $N_{\text {ave }}$ and $T_{\text {ave }}(\mathrm{MeV})$, can be calculated according to:

$$
\mathrm{P}\left(\mathrm{E}_{v}\right)=1.6 \times 10^{-4} \sigma_{v \text {-nucleus }} \mathrm{T}_{\text {ave }} \frac{\rho_{\text {nuclei }}}{\rho} \mathrm{Q}\left(\mu \mathrm{Sv} \mathrm{cm}^{2}\right)
$$

$\mathrm{Q}$, the quality factor, for these high LET ions is, conservatively, taken to have the maximum value of 30 (ICRP 1991). The distribution of recoil energy over tissue volume due to the short $(<<1 \mathrm{~cm})$ ionization ranges of the recoil ions in tissue is taken to be uniform.

\section{Process C: Neutrino scattering from nucleons}

For $50 \leq \mathrm{E}_{v} \leq 200 \mathrm{MeV}$, Boehm and Vogel (1992) give values of the total cross section, $\sigma_{v \text {-nucleon }}$ for scattering of neutrinos from nucleons. Over this energy domain, this cross section is a monotonically-increasing function of energy and ranges from $4.0 \times 10^{-41}$ to $5.5 \mathrm{x}$ $10^{-40} \mathrm{~cm}^{2}$. The corresponding cross section for antineutrinos is approximately $1 / 2$ as large. $P\left(E_{v}\right)$ can be obtained from 


$$
\mathrm{P}\left(\mathrm{E}_{v}\right)=\sigma_{v \text {-nucleon }} \mathrm{F}_{\text {nucleon }}\left(\mathrm{T}_{\text {ave }}\right) \mathrm{R}\left(\mathrm{T}_{\text {ave }}\right) \rho_{\text {nucleons }}\left(\mu \mathrm{Sv} \mathrm{cm}^{2}\right)
$$

where $R\left(T_{\text {ave }}\right)$ is the range of protons in tissue having a kinetic energy of $T_{\text {ave }} \cdot R \equiv 15 \mathrm{~cm}$ if the proton range exceeds $15 \mathrm{~cm}$. The restriction $\mathrm{R} \leq 15 \mathrm{~cm}$ reflects the fact that the average neutrino will interact in the center of the tissue slab. $F_{\text {nucleon }}\left(T_{\text {ave }}\right)\left(\mu \mathrm{Sv} \mathrm{cm}{ }^{2}\right)$ is the fluence-to-dose equivalent conversion factor of Schopper et al. (1990) for nucleons of kinetic energy $\mathrm{T}_{\text {ave }}$, averaged over the equally probable recoils of protons and neutrons. The "straight-ahead approximation", which deposits the energy of the recoil in the same direction as the incident neutrino and overstates the forward-peaking of the recoils found at these energies, is used.

For $\mathrm{E}_{\mathrm{v}} \geq 0.5 \mathrm{GeV}$, the interaction of neutrinos produces a number of secondary particles. This number, the multiplicity $\mathrm{M}$, is a monotonically-increasing function of the center of mass energy, $\mathrm{E}_{\mathrm{CM}}$, of the collision. For $0.5<\mathrm{E}_{\mathrm{v}}<10^{4} \mathrm{GeV}, 1.66<\mathrm{E}_{\mathrm{CM}}<137 \mathrm{GeV}$. Over this domain of $\mathrm{E}_{\mathrm{CM}}$ values of M have been tabulated by the Particle Data Group (1992) for leptonic (electron-positron) and hadronic (proton-antiproton) collisions. Since in this process, the scattering is "semi-leptonic", the averages of these two sets of values of $\mathrm{M}$ are used. M increases from 2 to 20 over this domain of $E_{v}$. For these energies, $\sigma_{v-\text { nucleon }}$ (for neutrinos) and $\sigma_{\bar{v}-\text { nucleon }}$ (for antineutrinos) are given by the Particle Data Group (1992), with $E_{v}$ in $\mathrm{GeV}$, as

$$
\begin{aligned}
\sigma_{v \text {-nucleon }} & =6.7 \times 10^{-39} \mathrm{E}_{v}\left(\mathrm{~cm}^{2}\right) \\
\text { and } \quad \sigma_{v-\text { nucleon }} & =3.4 \times 10^{-39} \mathrm{E}_{v}\left(\mathrm{~cm}^{2}\right) .
\end{aligned}
$$

$P\left(E_{v}\right)$ is estimated also using the straight-ahead approximation:

$$
\mathrm{P}\left(\mathrm{E}_{v}\right)=\sigma_{v \text {-nucleon }} \mathrm{M}\left(\mathrm{E}_{\mathrm{CM}}\right) \mathrm{F}_{\pi}\left\{\mathrm{T}_{\text {ave }} / \mathrm{M}\left(\mathrm{E}_{\mathrm{CM}}\right)\right\} \mathrm{R} \rho_{\text {nucleon }}\left(\mu \mathrm{Sv} \mathrm{cm}^{2}\right),
$$


where $F_{\pi}\left\{T_{a v e} / M\left(E_{C M}\right)\right\}$ is the fluence-to-dose equivalent conversion factor of Schopper et al. (1990) for positive pions $\left(\pi^{+1} s\right)$ at the average secondary particle energy. Thus, equal sharing of $\mathrm{T}_{\text {ave }}$ over the $\mathrm{M}$ secondary particles is assumed. The choice of $\pi^{+' s}$ to represent all of the secondary particles is conservative; alternative production of leptons (e.g., muons or electrons) would reduce the dose equivalent. This procedure benefits from the lack of strong dependence of $\mathrm{F}$ on hadron type or energy. $\mathrm{R}$ is again taken to be $15 \mathrm{~cm}$ to reflect the occurrence of the average interaction in the center of the tissue slab. The process is regarded as "flavor-blind", affecting all three flavors in the same manner. This formulation is most valid for high energy neutrinos encountered by the tissue slab in vacuum. Neutrinos emerging from a thick layer of material (e.g., earth shielding) and incident on the tissue slab might result in a slightly higher dose equivalent since the tissue slab would also be exposed to secondary particles, especially muons, produced by neutrinos interacting in the shielding material considerable distances "upstream" of the tissue slab. However, it is believed that the choice of the straight-ahead approximation as well as the selection of $\pi^{+1} s$ as the representative secondary particles more than compensate for this phenomena.

Fig. 1 plots $\mathrm{P}\left(\mathrm{E}_{\psi}\right)$ for the three processes for neutrinos (as distinct from antineutrinos). For process $\mathrm{A}$, this is done specifically for $\nu_{\mu}$ 's.

\section{APPliCATIONS TO SPECIFIC CALCULATIONS}

\section{$\underline{\text { Solar neutrinos }}$}

Bahcall (1989) has elegantly described the current understanding of the neutrinos emitted by the Sun. He gives the predicted total neutrino flux density of these neutrinos at the Earth of $6.6 \times 10^{10} \mathrm{~cm}^{-2} \mathrm{~s}^{-1}$. These neutrinos are all $\nu_{\mathrm{e}}$ 's. The dominant process by which the Sun emits neutrinos is that in which two protons interact to produce ${ }^{2} \mathrm{H}$ ("pp neutrinos"). This process results in a flux density on Earth of approximately $6 \times 10^{10} \mathrm{~cm}^{-2} \mathrm{~s}^{-1}$ and has a maximum of $\mathrm{E}_{\mathrm{v}}$ of approximately $0.42 \mathrm{MeV}$. The neutrinos produced in the part of the 
thermonuclear reaction chain involving ${ }^{8} \mathrm{~B}$ ("8 $\mathrm{B}$ neutrinos") have a maximum of $\mathrm{E}_{\mathrm{v}}$ of approximately $15 \mathrm{MeV}$. At Earth, the flux density of these neutrinos is approximately $5.8 \times 10^{6}$ $\mathrm{cm}^{-2} \mathrm{~s}^{-1}$. One can calculate the dose equivalent due to the solar neutrinos, assuming they all reach the Earth ${ }^{\S}$. For the pp neutrinos, Process A dominates, especially when one corrects the values in Fig. 1 to $v_{\mathrm{e}}$ 's (instead of $\nu_{\mu}$ 's) to obtain $\mathrm{P}=2.9 \times 10^{-26} \mu \mathrm{Sv} \mathrm{cm}^{2}$, assuming the maximum neutrino energy. Inserting the flux density, a dose equivalent rate, $\mathrm{dH} / \mathrm{dt}=1.7 \times 10^{-15} \mu \mathrm{Sv} \mathrm{s}^{-1}=5.5 \times 10^{-8} \mu \mathrm{Sv} \mathrm{y^{-1 }}$ is calculated. For the ${ }^{8} \mathrm{~B}$ neutrinos, both Processes A and B contribute. Assuming all neutrinos to have the maximum neutrino energy, Process $\mathrm{A}$ gives a value of $\mathrm{P}=7.2 \times 10^{-23} \mu \mathrm{Sv} \mathrm{cm}^{2}$ while for Process $\mathrm{B}, \mathrm{P}=1.0 \times 10^{-22} \mu \mathrm{Sv}$ $\mathrm{cm}^{2}$. Thus, the total is $\mathrm{P}=1.72 \times 10^{-22} \mu \mathrm{Sv} \mathrm{cm}{ }^{2}$ resulting in $\mathrm{dH} / \mathrm{dt}=1.0 \times 10^{-15} \mu \mathrm{Sv} \mathrm{s}^{-1}=$ $3.1 \times 10^{-8} \mu \mathrm{Sv} \mathrm{y}^{-1}$. Thus, for all solar neutrinos, $\mathrm{dH} / \mathrm{dt}$ is approximately $10^{-7} \mu \mathrm{Sv} \mathrm{y}^{-1}$ at the Earth.

\section{Estimate for the Fermilab Neutrinos at the Main Injector Project (NuMI)}

The U. S. Department of Energy is considering the construction of a new beam of neutrinos at Fermilab which would be directed through the crust of the Earth over a distance of about $800 \mathrm{~km}$ toward an existing proton decay experiment at Soudan, Minnesota. The goal is to see if any of the $\nu_{\mu}$ 's become $\nu_{e}$ 's or $\nu_{\tau}$ 's over their flight and, if so, demonstrate that neutrinos have a small, nonzero mass (Boehm and Vogel 1992). The designed beam of $v_{\mu}{ }^{\prime}$ s has an average energy of $13 \mathrm{GeV}$ so that only Process $\mathrm{C}$ is important. Calculations of the flux density of neutrinos in the center of this beam as a function of distance from the production target have been performed. Using eqn (9) which yields $P\left(E_{v}\right)=6 \times 10^{-15} \mu S v \mathrm{~cm}^{2}$, the dose equivalent rates shown in Table 1 are calculated. 


\section{EXTINCTION OF SPECIES}

J. Collar (1996) has presented a hypothesis that the final stages of stellar collapse could have led to the extinction of some species on earth due to the interaction of the neutrinos produced by these cosmic events. This theory has appeared, sometimes sensationally, in public media $^{\dagger}$. Collar observed that the neutrinos emitted by such events would be in the energy regime dominated by Process B. Noting the high LET of the recoiling nuclei, Collar concluded that these neutrinos could have caused the mass extinctions known from paleontology. Collar calculated the spectrum of neutrinos and determined their fluence as a function of distance from the collapse. Assuming tissue to be $\mathrm{C}_{4} \mathrm{H}_{40} \mathrm{O}_{17} \mathrm{~N}$, he calculated an average absorbed dose due to one of these collapses of $10^{-8} \mathrm{~Gy}$ at $3.09 \times 10^{13} \mathrm{~km}$ (one parsec or 3.26 light years). Given the chosen tissue composition, oxygen recoils would be the most damaging because of their prevalence, the enhanced $\sigma_{\text {nucleus }}$ due to the $\mathrm{N}^{2}$ factor in eqn (4), and their high LET $(\approx 257$ $\left.\mathrm{keV} \mu \mathrm{m}^{-1}\right)$. Collar deduced that as many as 4 to 12 malignant foci $\mathrm{kg}^{-1}$ in tissue on earth would occur due to collapses that might happen every 30 to $100 \mathrm{Myr}$. This result is based upon an estimate that the average recoil results in interactions with 3.6 nucleosomes out of $3 \times 10^{7}$ per cell nucleus and assumes that only one hit is required to result in malignancy. He concluded that large mass species, of relatively low populations, might have sufficient members killed by the tumors to result in nonviable populations. Smaller, more populous organisms might survive.

Several questions have been raised concerning this hypothesis (Cossairt and Marshall 1997). Collar cites results principally from in vitro studies. The connection of results from such studies to in vivo conditions with respect to specific end points presents problems that were not considered by Collar. Such problems have been discussed, e.g., by Turner and Fry (1994). References cited by Collar express conclusions, with suitable caveats, that unique and important biological effects may result from high (LET) radiation. The caveats, though not quoted by Collar, are important as exemplified by Goodhead (1988) who states, "Further understanding of these questions could lead, in future (sic), to substantial increases or 
decreases in estimations of risk." Recent studies that better define the boundaries of the effects of high LET radiation now exist (see reference list).

Collar has referred to "effectively infinite" values of relative biological effectiveness ( $\mathrm{RBE})$. One can estimate a value of $\mathrm{RBE}$ for the radiation damage due to the recoils for fatal cancer. An established measure of risk for Homo sapiens exposed to photons is $5 \times 10^{-2}$ latent fatal cancers $\mathrm{Gy}^{-1}$ (ICRP 1991). If the absorbed dose of $10^{-8} \mathrm{~Gy}$ due to a stellar collapse were due to photons, the expected incidence rate of fatal cancers would be $5 \times 10^{-10}$. For reference, humans are subject to a lifetime cancer mortality rate of about $20 \%$ (Cember 1983). It is thus unlikely that an increment of, say, one per cent in this mortality rate would result in extinction. For $10^{-8} \mathrm{~Gy}$ due to the neutrino recoils to result in a fatal incidence rate of 0.01 (one per cent), the RBE would have to be approximately $2 \times 10^{7}$. Accepted RBE values assigned for all end points inclusive of cancer induction, do not exceed approximately 200 (NCRP 1990). Specifically, values determined in recent work involving high LET ions are also bounded by this value, which is far smaller than needed to support Collar's conclusion (Scholz and Kraft 1994, Jenner et al. 1994, Kiefer et al. 1994, Edwards et al. 1994, and Barendsen et al. 1994).

\section{CONCLUSION}

Methods have been presented for calculating the dose equivalent due to neutrinos spanning a large range of energies. It is found that neutrinos produced by the Sun and presentday particle accelerators produce inconsequential dose equivalent rates. Examining recent calculations concerning neutrinos incident upon the Earth due to stellar collapse, it is concluded that it is highly unlikely that these neutrinos caused the mass extinctions of species found in the paleontological record. The authors would like to thank L. Belkora, D. Boehnlein, A. Elwyn, and K. Vaziri for their very helpful comments. 


\section{REFERENCES}

Bahcall, J. Neutrino astrophysics. New York: Cambridge University Press; 1989.

Barendsen, G. W. RBE-LET relationships for DNA lesions and different types of cellular damage. Rad. Prot. Dos. 52:359-362; 1994.

Boehm, F.; Vogel, P. Physics of massive neutrinos. $2^{\text {nd }}$ Ed. New York: Cambridge University Press; 1992.

Cember, H. Introduction to health physics New York: Pergamon Press; 1983: 184.

Collar, J. Biological effects of stellar collapse neutrinos. Phys. Rev. Lett. 76:999-1002; 1996.

Cossairt, J. D.; Marshall, E. T. Comment on "Biological effects of stellar collapse neutrinos". Phys. Rev. Lett. in press.

Drukier, A.; Stodolsky, L. Principles and applications of a neutral-current detector for neutrino physics and astronomy. Phys. Rev. D30:2295-2309; 1984.

Edwards, A. A.; Finnon, P.; Moquet, J. E.; Lloyd, D. C.; Darroudi, F.; Natarajan, A. T. The effectiveness of high energy neon ions in producing chromosomal aberrations in human lymphocytes. Rad. Prot. Dos. 52:299-303; 1994.

Goodhead, D. H. Spatial and temporal distribution of energy. Health Phys. 55:231-240; 1988. 
ICRP. 1990 recommendations of the International Commission on Radiological Protection. New York: Pergamon Press; ICRP Publication 60; 1991.

ICRP. Report of the task group on reference man. Oxford: Pergamon Press; ICRP Report 23; 1975.

Jenner, T. J.; de Lara, C. M.; Stevens, D. L.; Burns, N. A.; O'Neill, P. The induction of DNA double strand breaks in V79-4 cells by $\gamma$ and $\alpha$ radiations--complexity of damage. Rad. Prot. Dos. 52:289-293; 1994.

Kiefer, J; Ikpeme, S.; Akpa, T. C.; Weber, K. J. DNA double strand break induction by very heavy ions: dependence on physical parameters. Rad. Prot. Dos. 52:295-298; 1994.

Langacker, P.; Erler, J. Standard model of electroweak interactions. Phys. Rev. D54: 85-93; 1996.

NCRP. The relative biological effectiveness of radiations of different quality. Washington: NCRP Report 104; 1990.

Particle Data Group. Review of particle properties. Phys. Rev. D45:S1-S584; 1992.

Scholz, M.; Kraft, G. Calculation of heavy ion inactivation probabilities based on track structure, $\mathrm{x}$ ray sensitivity and target size. Rad. Prot. Dos. 52:29-33; 1994.

Schopper, H. , ed. Shielding against high energy radiation; Landolt-Börnstein numerical data and functional relationships; science and technology, new series; group I: nuclear and particle physics volume II. Heidelberg: Springer-Verlag; 1990. 
Turner, J. E.; Fry, R. J. M. High LET radiation carcinogenesis: what do we know and what do we need to know? Rad. Prot. Dos. 52:189-196; 1994. 


\section{FOOTNOTES}

"This work was performed at the Fermi National Accelerator Laboratory under contract DE-AC0276CH03000 with the U. S. Department of Energy.

$\S$ To date, experiments have found the flux densities of $v_{\mathrm{e}}$ 's to be less than these predicted values. This is the famous "solar neutrino problem" (see Boehm and Vogel 1992 and Bahcall 1989).

†These are exemplified by The Boston Globe, January 11, 1996, p. 13, The New York Times, January 23, 1996, p. B8, and Scientific American, April 1996, p. 20. 
Table 1 Results of calculations of dose equivalent for the proposed NuMI neutrino beam at Fermilab.

\begin{tabular}{ccc}
\hline $\begin{array}{c}\text { Distance } \\
\text { from Target }\end{array}$ & $\begin{array}{c}\text { Neutrino } \\
\text { Flux Density }\end{array}$ & $\begin{array}{c}\text { Dose } \\
\text { Equivalent } \\
\text { Rate }\end{array}$ \\
\hline $\mathbf{( k m )}$ & $\left(\mathbf{c m}^{-2} \mathbf{y r}^{-1}\right)$ & $\left(\mu \mathbf{S v ~ \mathbf { y r } ^ { - 1 } )}\right.$ \\
\hline 1 & $1.2 \times 10^{13}$ & $6.8 \times 10^{-2}$ \\
90 & $2.3 \times 10^{9}$ & $1.4 \times 10^{-5}$ \\
550 & $7.0 \times 10^{7}$ & $4.2 \times 10^{-7}$ \\
730 & $3.5 \times 10^{7}$ & $2.1 \times 10^{-7}$ \\
\hline
\end{tabular}




\section{FIGURE CAPTION}

Figure 1 Values of $\mathrm{P}\left(\mathrm{E}_{v}\right)$, the fluence-to-dose equivalent conversion factor, plotted as a function of neutrino energy, $E_{v}$ for the three processes described in the text. The results calculated for Process A for $\nu_{\mu}$ 's are plotted. Processes B and C are regarded as "flavor blind". Values for antineutrinos are smaller, see text. The symbols on the curves are at the coordinates where the calculations were performed. 


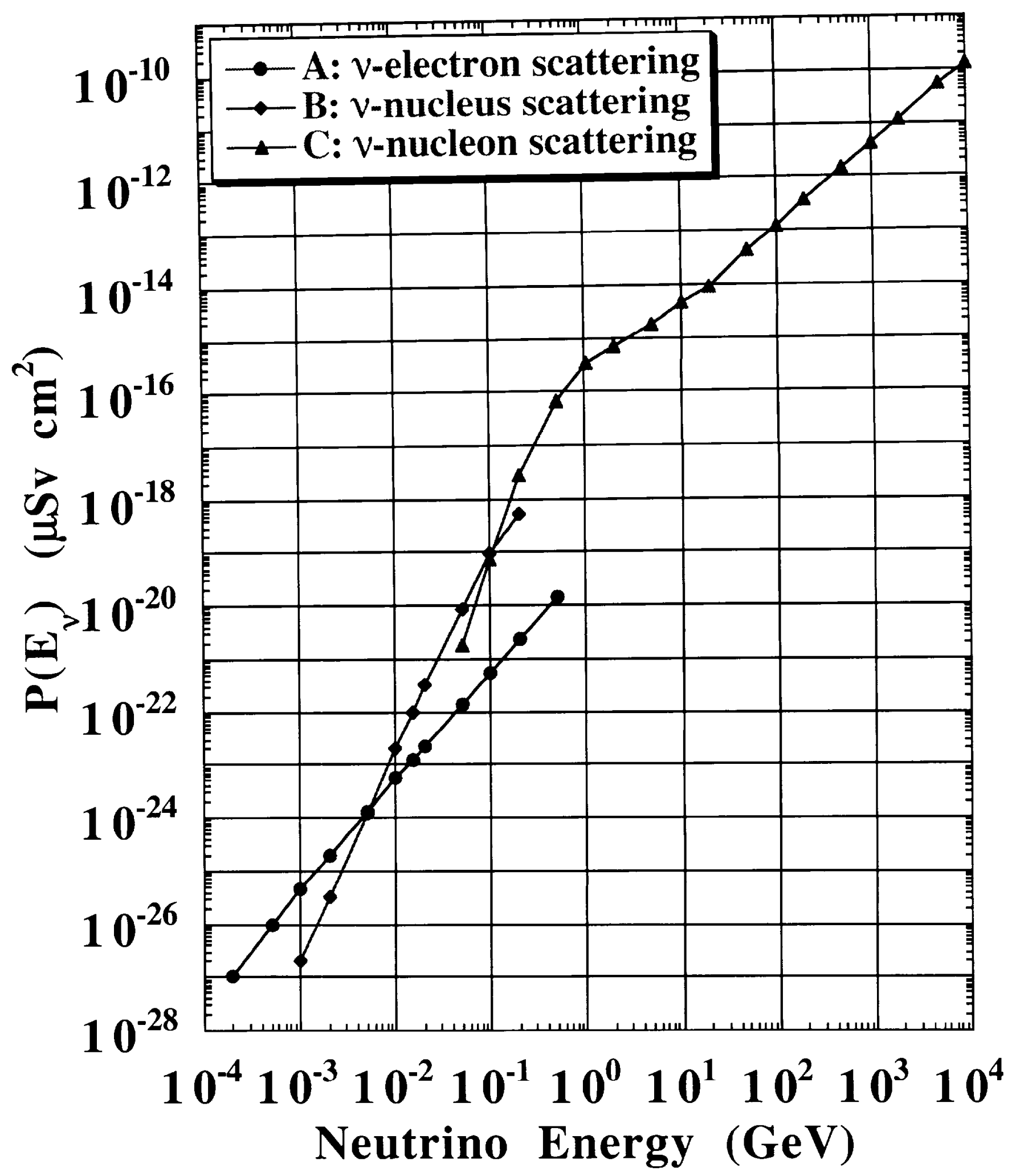

